\section{O7B.5 TRAJECTORIES OF PSYCHOSOCIAL WORKING CONDITIONS AS PREDICTORS OF LATER LIFE PHYSICAL FUNCTION}

${ }^{1}$ Charlotta Nilsen*, ${ }^{2}$ Ross Andel, ${ }^{1}$ Alexander Darin-Mattsson, ${ }^{1,3}$ Ingemar Kåreholt. ${ }^{1}$ Aging Research Center (Karolinska InstitutetStockholm University), Solna, Sweden; ${ }^{2}$ School of Aging Studies, University of South Florida, Tampa, USA; ${ }^{3}$ Institute of Gerontology, School of Health and Welfare, Aging Research Network - Jönköping (ARN-J), Jönköping University, Jönköping, Sweden

\subsection{6/OEM-2019-EPI.171}

Objectives The demographic shift towards an aging society has made it important to understand underlying life course trajectories of later life health and function. The aim was to investigate if psychosocial working conditions are associated with later life physical function.

Method Two individually linked longitudinal Swedish surveys were used $(n=803)$. A psychosocial job exposure matrix was used to measure psychosocial working conditions in the first occupation and at ages 25, 30, 35, 40, 45, and 50 - based on work history - and current occupation at baseline (1991). Physical function was measured in 2014. Random effects growth curve models were used to calculate within-person change. Random effects growth curve models were used to calculate intraindividual trajectories of working conditions, analyzed in relation to functional impairment with ordered logistic regression.

Results Having a more active job at baseline was associated with decreased odds of functional impairment in old age (OR 0.87, CI 0.76-0.99). Having a more high strain job at baseline was associated with increased odds of functional impairment in old age (OR 1.33, CI 1.04-1.70). Having a high starting point and upward trajectory of job strain throughout working life were associated with increased odds of functional impairment in old age (OR 3.16, CI 1.73-5.80).

Discussion Promoting a healthy workplace by reducing chronic stress and inducing intellectual stimulation, control, and personal growth, may not only improve the health of workers. It may also lower the cost of health and social care by improving health and function of the older population. Hence, investing in a healthy workplace should be seen as a double-win investment for society.

\section{B.6 MORTALITY BY SUICIDE IN THE SWISS NATIONAL COHORT (1990-2014): ANALYSIS ACCORDING TO OCCUPATION AND ECONOMIC ACTIVITY}

\begin{abstract}
${ }^{1}$ Irina Guseva Canu*, ${ }^{1}$ Nicolas Bovio, ${ }^{1}$ Zakia Mediouni, ${ }^{2}$ Murielle Bochud, ${ }^{1,3}$ Pascal Wild. ${ }^{1}$ Institute For Work And Health (IST), Lausanne, Switzerland; ${ }^{2}$ Institute of Social and Preventive Medicine (IUMSP), Lausanne, Switzreland; ${ }^{3}$ Institut National de recherche et de sécurité (INRS), Nancy, France
\end{abstract}

\subsection{6/OEM-2019-EPI.172}

Objective Given the known importance of work in our everyday lives, and growing economic and psychosocial pressure related to rapid changes in current working conditions, the link between occupation and suicide regains both academic and practical interest. Sex-specific mortality from suicide in Swiss working-age (18-65) population was investigated.

Methods The study sample comprised 5'834'618 participants of the Swiss National Cohort (SNC) (94'918'456 personyears). For every occupation and economic activity/industry (coded using international standard classifications) we computed directly age-standardized mortality rates (DSRs) using the age structure of the European population (2010) and standardized mortality ratios (SMRs) for suicide using Swiss population cause-specific mortality rates.

Results The highest DSRs were observed among unemployed/ job seeking group (52.94 per 100000 person-years based on 568 suicides), in agricultural, fishery and related male workers, and in health and social activities female workers. A consistent reduction in DSRs across three calendar periods, 1990-1998, 1999-2006, and 2007-2014 was observed in men. In female corporate managers, DSRs increased over the 2007-2014 period compared with 1999-2006. Unemployed/job seeking people, manufacturing labourers, personal care and related workers, and motor vehicle drivers of both sexes were identified at risk of suicide (SMR >1) compared to general working-age population. Moreover, an excess of suicide was observed among male material recording and transport clerks; nursing and midwife associated professionals; and agricultural workers as well as among female writers and performing artists.

Conclusions This study showed a distinctive pattern of suicide mortality between the two sexes and identified occupation groups at high and low risk of suicide among men and women. Sex-specific results need further investigation. In-depth analyses, taking into account the individual, social, and organizational characteristics of this population are necessary to understand the differences between and within protective and harmful occupations and to design targeted interventions aiming at suicide prevention among high-risk workers.

\section{Intervention Studies}

\section{C.2 DEVELOPING A MEASURING STRATEGY FOR MONITORING AND EVALUATION OF A NATIONAL OCCUPATIONAL HEALTH INTERVENTION PROGRAMME IN GREAT BRITAIN (GB)}

Yiqun Chen*, Andrew Curran. Health and Safety Executive, Buxton, UK

\subsection{6/OEM-2019-EPI.173}

The Health and Safety Executive (HSE) is the GB regulator for health and safety at work. The HSE Health and Work (H\&W) program designs and carries out a wide range of interventions; including inspection, enforcement and other regulatory activities as well as prevention; targeting priority health conditions in high-risk sectors. It is anticipated that long-term, sustainable and coordinated actions developed as part of the program will over time improve awareness, behaviors, control of exposures, and, as a result, prevent workrelated ill health in GB workforce.

An HSE Measuring Strategy, together with measurement framework and principles, has been developed. The measurement framework draws together data systems, covering Attitudes (A), Behaviors (B), Control of exposures (C), and Disease and work-related ill health reduction (D), based on a simple model to provide evidence required for evaluating the short, medium and long term impacts of the large scale and complex H\&W program on the GB health and safety system. The Strategy gives a new focus on measuring behavioral changes and risk reductions; and emphasizes longitudinal measurement designs to assess progress over time. 